
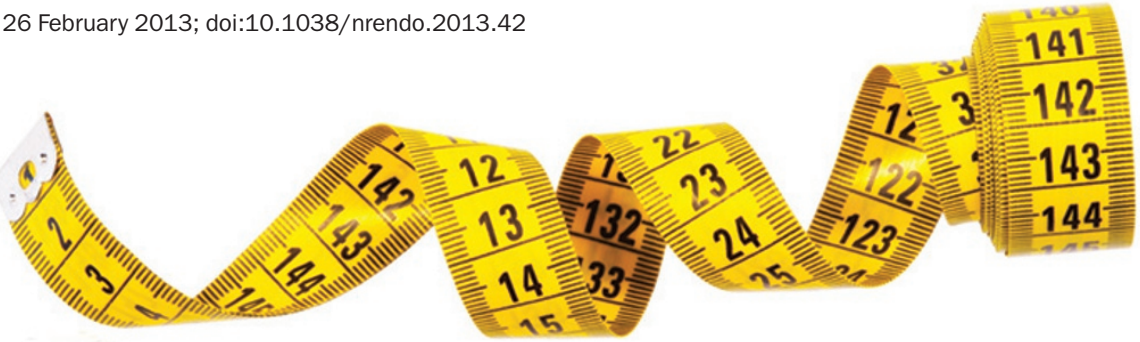

\title{
Teaching an old drug new tricks-amlexanox targets inflammation to improve metabolic dysfunction
}

A mlexanox, an anti-inflammatory medication used to treat mouth ulcers and asthma, could have some benefit for the treatment of obesity and type 2 diabetes mellitus, a study published in Nature Medicine shows. Moreover, the findings shed light on how energy balance is regulated by inflammation.

Myriad studies in animals and in humans implicate inflammation in the development of obesity-associated insulin resistance. "We have been studying inflammatory links between obesity and insulin resistance for some time, with a particular focus on macrophages in adipose tissue," recounts senior study investigator Alan R. Saltiel (University of Michigan, Ann Arbor, MI, USA). "Our discovery of macrophage subtype switching during obesity led us to search for genes involved in this process, and we learned that expression of the noncanonical I $\mathrm{B}$ kinases IKK- $\varepsilon$ and TBK1 was elevated
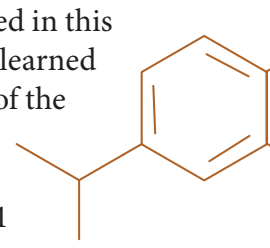
$\left.\right|_{0} ^{1}$ in obesity and insulin resistance." In a previously published study, Saltiel and colleagues found that mice lacking IKK- $\varepsilon$ who were fed a high-fat diet gained less weight and developed less insulin resistance and fatty liver disease than control mice. This finding prompted the investigators to search for chemical inhibitors of the two kinases.

Saltiel's team screened 150,000 chemical compounds, including drugs already on the market and chemicals derived from natural sources, and identified amlexanox, an approved small-molecule therapeutic, as a high-affinity inhibitor of IKK- $\varepsilon$. The researchers then fed mice a high-fat diet and treated one group with amlexanox. Whereas untreated mice became obese, animals treated with amlexanox maintained their body weight. In a second experiment, Saltiel and co-workers administered amlexanox to mice that were already obese: to leptin-deficient $o b / o b$ mice, to test the drug's effects on genetic obesity, and to diet-induced obese mice. Body weights of both groups returned to almost normal, and the weight loss was maintained for as long as the drug was administered.

To elucidate the mechanism underlying the effects of amlexanox, the investigators performed a detailed assessment of metabolic parameters, including insulin sensitivity (by hyperinsulinaemic euglycaemic clamp studies), energy expenditure (by indirect calorimetry), phosphorylation patterns and in vivo and in vitro changes in energy disposition. "The answer is that amlexanox restores the ability of mice to adapt to increased caloric intake by subsequently increasing energy expenditure through<smiles>Cc1cc2cnc1CC2</smiles><smiles>Nc1ncccc1C(=O)O</smiles>
thermogenesis," explains Saltiel. Importantly, the findings by Saltiel and colleagues further elucidate the $\mathrm{OH}$ mechanisms through which inflammation modulates energy balance. "We suspect that obesity-related inflammation produces not only insulin resistance but also catecholamine resistance, in such a way that fat cells (and possibly liver cells) become metabolically inflexible and are unable to respond to either anabolic or catabolic signals," says Saltiel. In other words, the results suggest that IKK- $\varepsilon$ and TBK1 are part of a counterinflammatory process that sustains energy storage in the presence of insulin resistance. The authors speculate that the reduced inflammation observed with amlexanox treatment is an indirect effect of improved metabolic disease or, perhaps, the result of elimination of a feedback loop that maintains inflammation at low levels.

Amlexanox has been in clinical use for more than 25 years, and the possibility that it might be repurposed for the treatment of obesity or type 2 diabetes mellitus is bound to generate a vast amount of interest. "The specific action of amlexanox is remarkable," comments Michael I. Goran (Keck School of Medicine, Childhood Obesity Research Center, University of Southern California, Los Angeles, CA, USA), who is not affiliated with the study. "This very targeted approach to reduce inflammation might cause less serious adverse effects compared with blunting a whole arm of the inflammation pathway," points out Goran, "and could potentially avoid a trade-off between improving metabolic health and the potential adverse effect of immunocompetence that can arise when using general anti-inflammatories to address adipose tissue inflammation."

Saltiel and his team plan to build on their findings and elucidate the mechanisms underlying IKK- $\varepsilon$ and TBK1 activity. "We have some leads on the kinase substrates and are also looking at gene expression patterns over time in mice treated with amlexanox," says Saltiel. "At the same time, we are planning clinical trials in patients with obesity and type 2 diabetes mellitus and nonalcoholic fatty liver disease, which we hope to start soon."

The outcome of these human studies will determine the actual impact that the findings by Saltiel and co-workers will have on clinical practice. Given that data on long-term drug effects in large patient populations are lacking, enthusiasm for this old drug's new tricks should be coupled with caution. "As this drug is capable of modulating immune activation, it is critical to determine what unintended consequences treatment could have in the face of pathogen exposure," concludes Goran.

\section{Linda Koch}

Original article Reilly, S. M. et al. An inhibitor of the protein kinases TBK1 and IKK- $\varepsilon$ improves obesity-related metabolic dysfunctions in mice. Nat. Med. doi:10.1038/nm.3082 